Methods We retrospectively reviewed the clinical records of 20 ocular syphilis patients presenting to Shanghai Clinical Center who completed CSF examination during the period from Jan 2011 to Nov 2012. Chief complaint, demographic data, syphilis serology, HIV serology and CSF examination results were collected. Factors that influenced the abnormal CSF results were explored.

Results The interval between ocular disease onset and the first visit at our hospital ranged from 2 weeks to 20 years (median 4 months). The mean age was 52.4 years, 14 patients were male and 6 were female. All patients tested negative for HIV infection. Median serum TRUST titer was 1:16 (ranged from 1 to 512 ). 14 (70\%) of the 20 patients had abnormal CSF (elevated WBC or protein levels or reactive VDRL results). Of which, 11(55\%) had elevated WBC cells, $11(55 \%)$ had elevated protein level, $8(40 \%)$ had reactive VDRL results. Patients with higher serum TRUST titer $(\geq 8)$ were more likely to have an abnormal CSF results.

Conclusion Unlike most of the ocular syphilis reports in recent years, the co-infection of syphilis and HIV was less commonly observed in China. Most (70\%) of these HIV-Negative patients had an elevated CSF WBC or protein level even with a moderate serum TRUST titer. Reactive CSF VDRL rate is not very high in our study.

\section{P2.194 OUALITY IMPROVEMENT: COTRIM PROPHYLAXIS IN HIV POSITIVE ADULTS ON ANTIRETROVIRAL TREATMENT}

doi:10.1136/sextrans-2013-051184.0458

A Casseus, J A Averty, F Laventure. Centre de Sante de Cerca-la-Source, Cerca-laSource, Haiti

Background At "Centre de Santé de Cerca-La-Source", a health clinic in rural Haiti, it was remarked that despite best efforts a number of patients with low CD4 counts and on ART have not been given access to cotrimoxazole prophylaxis. This in turn leads to increased vulnerability to opportunistic infections potentially raising the morbidity and mortality rates in the area.

Methods The tools and methodology of Quality Improvement in HIV care were used to quantify the problem. A prospective interventional study was put in place for a 6 month period: Apr-Sept 2012. During the 3 semesters prior, the percentage of patients receiving the prophylaxis was: 47\%, 46\%, 46\% (Oct 2010-Mar 2011, Apr 2011Sept 2011, and Oct 2011-Mar 2012 respectively). This project aimed to reach $90 \%$. The assigned team through brainstorming techniques and a modified Ishikawa diagram identified causes and two main interventions dubbed: validation and synchronisation.

Results Measurement was taken after full synchronisation between EMR (Electronic Medical Record) with the pharmacy data and patient hardcopy files, all done after each intervention within a period of two weeks past each. From the $1^{\text {st }}$ intervention: an increase of $14.84 \%$ of the indicator and after the second increase of $19 \%$, leading finally to $83.23 \%$. Final evaluation of the indicator at the end of the period after continued application of the interventions showed $93.55 \%$, a little over the targeted $90 \%$ objective.

Conclusion This lead to greater care in verification of data integrity within our system. Patient care is automatically improved once there is betterment of a system in place to monitor what has been done and what needs to be done. Furthermore, prophylaxis reduces the mortality rate and the probability of ailments that will require hospitalisation of the patients. In a resource limited system, the discordance between EMR and reality can be quite enormous.

\section{P2.195 A CASE SERIES OF TENOFOVIR INDUCED NEPHROTOXICITY}

doi:10.1136/sextrans-2013-051184.0459

S L Kay, K Hall, K Radcliffe. Queen Elizabeth Hospital, Birmingham, UK

Introduction Tenofovir (TDF) is a commonly used nucleotide reverse transcriptase inhibitor, effective in the management of
HIV-positive individuals and chronic Hepatitis B infection. We report two cases that highlight the rare but significant nephrotoxic adverse effects of TDF on mitochondrial-rich proximal renal tubular cells, causing acute and chronic kidney impairment. We also show that removal of TDF results in partial improvement of the renal dysfunction.

Case reports The first case describes a patient with long standing HIV who develops Fanconi syndrome, nephrogenic diabetes insipidus, acute tubular necrosis and tubulointerstitial nephritis, three years after TDF initiation. The second case reports a patient who developed acute severe kidney injury, requiring management with emergency haemodialysis, two weeks after TDF was initiated. In both cases renal biopsy showed extensive tubular injury and histology consistent with TDF induced renal injury. Both individuals had improved renal function following TDF cessation, but ongoing chronic kidney disease.

Discussion Nephrotoxicity has been demonstrated in $17-22 \%$ of TDF-treated patients and occurs due to TDF accumulation in cell membrane transporters within proximal tubular cells, where it targets the mitochondria. TDF-related nephrotoxicty presents in a number of ways including proximal tubular dysfunction, acute and chronic kidney injury and a partial or complete Fanconi syndrome. Histological findings most commonly show proximal tubular injury. TDF specific histology is related to prominent eosinophillic intracytoplasmic inclusions, representing giant mitochondria.

Conclusion TDF is a popular choice of antiretroviral therapy due to its efficacy, low side effect profile and use in monotherapy regimes. These cases highlight the potentially life-threatening complications of TDF-related nephrotoxicty. It also shows the importance of renal function monitoring and the withdrawal of TDF to prevent chronic renal impairment.

\section{P2.196 COMPARATIVE DETECTION OF CHLAMYDIA TRACHOMATIS IN CLINICAL SPECIMENS OBTAINED FROM GENITAL AND EXTRAGENITAL SITES}

doi:10.1136/sextrans-2013-051184.0460

V A Feodorova, ${ }^{1,2}$ S S Konnova, ${ }^{3} \mathrm{~A}$ V Kazantsev, ${ }^{3} \mathrm{E}$ S Sultanakhmedov, ${ }^{1} \mathrm{~T}$ I Polyanina, ${ }^{3} \mathrm{~S} R$ Utz, ${ }^{1,3} \mathrm{~A}$ M Lyapina, ${ }^{3} \mathrm{E}$ P Lyapina, ${ }^{3} \mathrm{E}$ P Lyapina, ${ }^{4} \mathrm{~V}$ L Motin. ${ }^{\circ}$ Saratov State Academic Veterinary Institute, Saratov, Russian Federation; ${ }^{2}$ Saratov State University, Saratov, Russian Federation; ${ }^{3}$ Saratov State Medical University, Saratov, Russian Federation; ${ }^{4}$ University of Texas Medical Branch, Galveston, TX, United States

Background Diagnosisof urogenital Chlamydia infection can be a challenging task in some cases due to difficulties in detection of the pathogen at the anatomical sites of humans. The goal of this study was to compare the efficiency of detection of C. trachomatis in samples obtained from different anatomical sites of patients.

Methods Clinical specimens from both genital (urethra, cervix) and extragenital sites (pharynx, conjunctive) were collected from patients with either urogenital (Group 1, $n=7$ ) or extragenital Chlamydia infection (Group 2, $\mathrm{n}=2$ ). Presence of Chlamydia in these samples was determined by PCR with primers to the C. trachomatis cryptic plasmid genes, as well as by commercial direct fluorescent test (DFT) and dot-ELISA.

Results Among Group 1 patients, 85.7\% urethral \& cervical, 75\% conjunctive and $50 \%$ pharyngeal samples were positive in PCR. Notably, DNA isolated from specimens of one individual showed negative result to Chlamydia at anatomical site, yet displayed a positive reaction in samples taken from both extragenital sites. Moreover, $100 \%$ of samples obtained from all different sites reacted positively in dot-ELISA for each patient. Six out of seven patients were positive for Chlamydia by DFT assay in samples acquired from the genital site (urethral \& cervical scrapes). Group 2 patients gave $100 \%$ positive response in PCR tests with DNA from genital and at least from one of the extragenital sites. 